\title{
Freezing of compact random heteropolymers with correlated sequence fluctuations
}

\section{Citation}

Chakraborty, Arup K., Eugene I. Shakhnovich, and Vijay S. Pande. 1998. "Freezing of Compact Random Heteropolymers with Correlated Sequence Fluctuations." The Journal of Chemical Physics108 (4): 1683-87. https://doi.org/10.1063/1.475539.

\section{Permanent link}

http://nrs.harvard.edu/urn-3:HUL.InstRepos:41534246

\section{Terms of Use}

This article was downloaded from Harvard University's DASH repository, and is made available under the terms and conditions applicable to Other Posted Material, as set forth at http:// nrs.harvard.edu/urn-3:HUL.InstRepos:dash.current.terms-of-use\#LAA

\section{Share Your Story}

The Harvard community has made this article openly available.

Please share how this access benefits you. Submit a story.

Accessibility 


\title{
Freezing of compact random heteropolymers with correlated sequence fluctuations
}

\author{
Arup K. Chakraborty \\ Department of Chemical Engineering and Department of Chemistry, University of California, Berkeley, \\ California 94720 \\ Eugene I. Shakhnovich \\ Department of Chemistry, Harvard University, Cambridge, Massachusetts 02138
}

Vijay S. Pande

Department of Physics, University of California, Berkeley, California 94720

(Received 18 August 1997; accepted 16 October 1997)

\begin{abstract}
Random heteropolymers (RHPs) with uncorrelated sequence fluctuations on the segmental scale can undergo a transition wherein, below a certain temperature, the thermodynamics is determined by a few dominant conformations. We study this "freezing" transition for RHPs with correlated sequence fluctuations. Specifically, we apply our theory to the case where the correlations decay with a single correlation length; a pragmatically realizable example is provided by random block copolymers. Our results show that the temperature at which freezing occurs grows with the block length of such polymers. Freezing also occurs on the scale of the correlation length, thus making experimental observation of this phenomenon (a consequence of frustration coupled with quenched disorder) more accessible. The results are rationalized on physical grounds. (C) 1998 American Institute of Physics. [S0021-9606(98)51604-2]
\end{abstract}

\section{INTRODUCTION}

Random heteropolymers (RHPs) are multicomponent polymers with a disordered sequence distribution. In recent years, there has been much interest in understanding the behavior of these polymers due to a variety of reasons. Like any multicomponent polymeric system, the energy scales characterizing interactions between different types of RHP segments can be different, thus leading to competing interactions. In addition, RHPs constitute a special class of soft matter wherein a quenched disorder (the sequence distribution) is carried by the fluid whose statistical properties are of interest. This combination of quenched disorder and competing interactions leads to a special type of frustration which, in turn, lends these materials many unusual properties. One such feature is that, below a certain temperature, RHPs undergo a transition wherein, instead of sampling a multitude of conformations, only a few dominant conformations determine the thermodynamics. We shall succinctly refer to this transition as freezing (not to be confused with liquid-solid transitions). Such a transition is of fundamental interest, and is akin to protein folding. Hence, much effort (e.g., Refs. 1-4) has been directed toward studying RHP physics with a view toward understanding protein folding.

Another motivation for studying RHP physics is the following. Nature mediates many specific functions through the action of biopolymers (such as proteins). Biopolymers are multifunctional, and furthermore, their sequences do not exhibit an ordered pattern. ${ }^{5,6}$ It is appealing to explore whether multifunctionality and quenched disorder are the generic physical features that nature exploits in order to mediate specific functions. RHPs can be prepared synthetically, and these polymers also embody multifunctionality and quenched disorder. Efforts have thus been expended at examining whether proper choice of the sequence statistics of synthetic RHPs can allow them to exhibit biomimetic behavior in certain contexts. ${ }^{78}$ Motivated by applications, the adsorption of RHPs from solution and their role as adhesion promoters have also been studied (e.g., Refs. 9-15).

In this paper, we focus on a very specific issue. Theoretical studies (e.g., Refs. 3,16-19) of the freezing transition of RHPs have all been concerned with situations where the disordered sequence distribution exhibits uncorrelated fluctuations. Here, we study the effect of correlated sequence fluctuations on the freezing transition. [Note that the microphase ordering of RHPs with correlated sequence fluctuations has been studied in the past (e.g., Ref. 20).] We develop the formalism generally, and then discuss results for the special case where the correlations decay with a single characteristic length. RHPs synthesized by randomly connecting blocks of monomers of the same type (oligomers or pre-polymers) provide an example. For this situation, we derive explicit relationships for the dependence of the temperature at which the freezing transition occurs on the block length. The results show that the manifestations of freezing should be easier to study experimentally for RHPs with sequence fluctuations characterized by a finite correlation length (compared to RHPs with uncorrelated sequence fluctuations).

The rest of the paper is organized as follows. In Sec. II, we describe the model and associated analysis. In Sec. III, the physical reasons that underlie the main results are discussed, and implications for experiments are noted. Finally, in Sec. IV, brief concluding remarks are offered.

\section{MODEL DEVELOPMENT AND THEORY}

For simplicity, we consider a RHP made up of two kinds of units $(A$ and $B)$. The Edwards Hamiltonian (on the segmental scale) for the problem of interest is 


$$
\begin{aligned}
-\beta H= & -\frac{3}{2 l} \int_{0}^{N} d n\left(\frac{d r}{d n}\right)^{2}-\frac{a}{2} \int_{0}^{N} d n \int_{0}^{N} d n^{\prime} \\
& \times \delta\left[r(n)-r\left(n^{\prime}\right)\right]+\frac{b}{2} \int_{0}^{N} d n \int_{0}^{N} d n^{\prime} \delta(r(n) \\
& \left.-r\left(n^{\prime}\right)\right) \theta(n) \theta\left(n^{\prime}\right),
\end{aligned}
$$

where $\beta$ is inverse temperature, $N$ is the chain length, $l$ is the statistical segment length, and $a$ and $b$ are defined as follows:

$$
\begin{aligned}
& a=\frac{V_{A A}+V_{B B}+2 V_{A B}}{4}, \\
& b=-\frac{V_{A A}+V_{B B}-2 V_{A B}}{4} .
\end{aligned}
$$

$V_{i j}$ are the energy scales characterizing the interactions between segments of type $i$ and $j$. $\theta$ is a stochastic variable that measures whether a given segment is of type $A$ or $B(\theta=+1$ if the segment is of type $A$, and equals -1 if the segment is of type $B$ ).

In writing Eq. (1), we have assumed that $V_{A A}=V_{B B}$. This does not affect any of the qualitative results since we are concerned with compact globular states. ${ }^{16}$ Equation (2) shows that the energy parameter, $b$, is closely related to the Flory parameter, $\chi_{F} ; \chi_{F}=2 b$.

In order to analyze this Hamiltonian, we first decouple the non-local term using the Hubbard-Stratonovich transform. The quenched average over sequence fluctuations is carried out using the replica trick. ${ }^{21}$ In our model, we represent correlated sequence fluctuations in the simplest possible way; viz., the distribution is taken to be Gaussian. The probability distribution characterizing sequence fluctuations is therefore taken to be

$$
\begin{aligned}
P[\delta \theta(n)] \propto & \exp \left[-\frac{1}{2} \int d n \int d n^{\prime} \delta \theta(n)\right. \\
& \left.\times \chi^{-1}\left(n-n^{\prime}\right) \delta \theta\left(n^{\prime}\right)\right]
\end{aligned}
$$

where $\chi\left(n-n^{\prime}\right)=\left\langle\delta \theta(n) \delta \theta\left(n^{\prime}\right)\right\rangle$, and determines the nature of the correlations that characterize the fluctuations.

After the Hubbard-Stratonovich transformation, and averaging over sequence fluctuations, the $m$-replica partition function may be written as

$$
\left\langle G^{m}\right\rangle=\iint D Q_{\alpha \beta} \exp \left[-E\left(Q_{\alpha \beta}\right)+S\left(Q_{\alpha \beta}\right)\right],
$$

where

$$
\begin{aligned}
E= & -\ln \Pi_{\alpha} \iint D \phi_{\alpha}(r) \\
& \times \exp \left[-\frac{1}{2} \sum_{\alpha \beta} \int d r \int d r^{\prime} \phi_{\alpha}(r) P_{\alpha \beta}\left(r-r^{\prime}\right) \phi_{\beta}\left(r^{\prime}\right)\right],
\end{aligned}
$$

$$
\begin{aligned}
& S= \ln \Pi_{\alpha} \iint D r_{\alpha}(n) \exp \left[-\frac{3}{2 l} \sum_{\alpha} \int d n\left(\frac{d r_{\alpha}}{d n}\right)^{2}\right] \\
& \times \Pi_{\alpha \beta} \delta\left[\int d n \int d n^{\prime} \delta\left[r_{\alpha}(n)-r\right)\right. \\
&\left.\quad \times \chi\left(n-n^{\prime}\right) \delta\left[r_{\beta}\left(n^{\prime}\right)-r^{\prime}\right]-Q_{\alpha \beta}\right] \\
& P_{\alpha \beta}\left(r-r^{\prime}\right)=\delta_{\alpha, \beta} \delta\left(r-r^{\prime}\right)-b Q_{\alpha \beta}\left(r-r^{\prime}\right), \\
& Q_{\alpha \beta}\left(r-r^{\prime}\right)=\int d n \int d n^{\prime} \delta\left(r_{\alpha}(n)-r\right) \chi\left(n-n^{\prime}\right) \\
& \times \delta\left(r_{\beta}\left(n^{\prime}\right)-r^{\prime}\right),
\end{aligned}
$$

where $\Phi$ is the Hubbard-Stratonovich field, $\alpha$ and $\beta$ are replica indices, and $Q_{\alpha \beta}$ is an order parameter that measures the correlated overlap between replicas. We may view $E$ to be the energy associated with a given overlap order parameter, and $S$ to be the entropy.

So far, the development follows that for sequence fluctuations with short-ranged correlations, except for the factor $\chi$ in the definition of the overlap order parameter. This correlation function contains all information regarding the correlated sequence fluctuations. On the segmental length scale, the correlation function may be written as

$$
\chi\left(n-n^{\prime}\right)=C_{1} \delta\left(n-n^{\prime}\right)+C \chi^{d}\left(n-n^{\prime}\right),
$$

where $C$ and $C_{1}$ are constants, and $\chi^{d}$ is the distinct part of the correlation function (for fluctuations with short-ranged correlations, $C \chi^{d}=0$.

In order to examine the essential physics, we consider the simplest possible type of correlated sequence fluctuations; viz., one wherein the distinct part of the correlation function decays with a single characteristic length, $\xi$. Physically, this corresponds to a RHP synthesized by randomly connecting oligomers (of length $\xi$ ) made up of either $A$ - or $B$-type segments: in other words, random block copolymers. Synthetic polymers of this sort may be easy to prepare for careful experimental studies of the phenomenon of freezing into a few dominant conformations. If we wish to examine properties on scales larger than this correlation length, the qualitative behavior of such a system will be reproduced by taking $\chi^{d}$ to be a step function with width $\xi$. In the following, we analyze how the freezing temperature changes with $\xi$. The qualitative results are expected to be true for arbitrary forms of $\chi^{d}$, as long as we interrogate the system on scales larger than the correlation length characterizing the sequence fluctuations. The analysis to follow would be inappropriate however, if the sequence fluctuations exhibited infiniteranged correlations ${ }^{22}$ or if $A$ - and $B$-type segments have an enhanced propensity to alternate within a correlation length.

Since we are looking at situations where the sequences are correlated on the scale $\xi$, we are interested in studying freezing on commensurate length scales (the length of the blocks in the random block copolymer example). This is so, because on shorter length scales we have homopolymers that cannot freeze. For the purpose of examining the system on this length scale, we need only consider the distinct part of 
the correlation function. Specifically, Eq. (5) must be evaluated with the distinct part of the correlation function taken to be

$$
\chi\left(n-n^{\prime}\right)=C W\left(\left|n-n^{\prime}\right|-\xi\right),
$$

where $W$ is the step function.

Substituting Eq. (7) into the definition of $Q_{\alpha \beta}$ [Eq. (5)], it becomes clear that the overlap order parameter measures overlap between replicas on the scale, $\xi$ (along the sequence). This is in contrast to RHPs with sequence fluctuations on the segmental scale where the relevant overlap is that between replicas on the scale of segments. For the physical problem at hand, the replica overlap is that between the blocks. On physical grounds, this formal result is transparent.

In order to compute the freezing temperature on this scale, we must renormalize Eq. (5) from the segmental scale to the scale, $\xi$. In other words, we must renormalize the computation of the energy and entropy in Eq. (5) to this scale. Let us begin with the computation of the energy. Since we wish to study the phenomenon of freezing, we must allow for the possibility of replica symmetry breaking (RSB), and then compute the Gaussian integral in Eq. (5) using the formula developed by Mezard and Parisi ${ }^{23}$ for the trace of the logarithm of hierarchical matrices. But first, in order to compute the energy on the appropriate scale, we must renormalize the energy parameter, $b$, defined on the segmental scale to the scale $\xi$. This is easily done since we have a compact state with constant density. Noting that there are $\xi^{2}$ possible segmental interactions in a spatial volume proportional to $\xi^{3 / 2}$, the renormalized energy scale becomes

$$
\bar{b}=b A \sqrt{\xi},
$$

where $\bar{b}$ is the renormalized energy scale, and $A$ is a constant that we shall not bother to specify since we are concerned only with the scaling of the freezing temperature with $\xi$.

We now need to introduce a RSB scheme. Since we are concerned here only with correlation functions of finite range, like for RHPs with uncorrelated sequence fluctuations, we introduce a one-step RSB scheme. (Simulations ${ }^{22,24}$ have provided evidence that such a REM-like picture ${ }^{25}$ is qualitatively correct as long as the correlations remain of finite range.) Within such a scheme, replicas are divided into groups (with $x_{0}$ being the number of replicas in a group). Replicas within a group overlap perfectly, while replicas in different groups do not overlap at all. The energy calculation is straightforward ${ }^{16}$ for this one-step RSB scheme, and the energy per block (after taking the replica limit) is found to be

$$
E=\frac{1}{2}\left[\frac{1}{x_{0}} \ln \left(\bar{b}-C \overline{b^{2}} \rho x_{0}\right)+\left(\frac{x_{0}-1}{x_{0}}\right) \ln \bar{b}\right],
$$

where $\rho$ is the density of the compact state (set by other chain parameters). $x_{0}$ acquires values between 0 and 1 , and has the usual physical meaning: ${ }^{25} x_{0}=1-\Sigma_{i} P_{i}^{2}$, where $P_{i}$ is the probability associated with conformation $i$ (on the scale $\xi)$. Thus, as usual, freezing into a few dominant conformations is signaled by this order parameter acquiring values less than unity.
Having computed the energy, we now proceed to calculate the entropy corresponding to Eq. (5) using the one-step RSB scheme. Keeping in mind that overlap is defined on the scale $\xi$, the entropy is calculated as follows. Consider a given replica. The first block is placed at a given position (measured on the scale $\xi$ ). The number of ways of placing the next block is proportional to the area of the surface enclosing the volume occupied by the first block. This is so because the first segment of the next block could emanate from any point on this surface. Thus, the number of ways of placing the second block is $D \xi ; D$ equals $4 \pi *$ (the number of ways of placing $\xi$ segments in the volume occupied by these segments in the compact state). For compact states, the chain conformational statistics are Gaussian, and hence the quantity multiplying $4 \pi$ in the preceding sentence is a constant related to chain flexibility. ${ }^{26}$ Therefore, $D$ is independent of $\xi$. Now noting that once the first replica assumes a particular conformation the remaining $\left(x_{0}-1\right)$ replicas in a group must be placed exactly the same way, that there are $N / \xi$ renormalized segments, and that there are $m / x_{0}$ groups, we find the entropy per renormalized segment to be (after taking the replica limit)

$$
S=\left(\frac{x_{0}-1}{x_{0}}\right) \ln (D \xi) .
$$

Combining Eqs. (9) and (10) we obtain the free energy density for a given overlap order parameter to be

$$
\begin{aligned}
f= & \frac{1}{2}\left[\frac{1}{x_{0}} \ln \left(\bar{b}-C \overline{b^{2}} \rho x_{0}\right)+\left(\frac{x_{0}-1}{x_{0}}\right) \ln \bar{b}\right] \\
& -\left(\frac{x_{0}-1}{x_{0}}\right) \ln (D \xi),
\end{aligned}
$$

where $f$ is the free energy density.

A mean-field solution is now obtained by extremizing Eq. (11) with respect to $x_{0}$. We find that $x_{0}$ satisfies

$$
\ln \left(1-C \bar{b} \rho x_{0}\right)+\frac{C \bar{b} \rho x_{0}}{1-C \bar{b} \rho x_{0}}=2 \ln (D \xi) .
$$

Equation (12) can be solved for $x_{0}$ implicitly. In order to see the scaling of the freezing temperature with $\xi$ (the essential result of interest), we solve Eq. (12) explicitly for $x_{0}$ in the limit of small values of $b$. We find that $x_{0}$ is of the form $T / T_{f}$, where $T$ is temperature, and the temperature $T_{f}$ below which the thermodynamics is determined by a few dominant conformations (on the scale $\xi$ ) is given by

$$
T_{f}=A b \sqrt{\frac{\xi}{2 \ln (D \xi)}} .
$$

Equation (13) is the result that we seek; the variation of the temperature at which freezing occurs with the length scale over which sequence fluctuations of the RHP are correlated.

Prior to discussing this result, for comparison, we derive a simple scaling relationship for the variation of the temperature at which microphase segregation occurs with $\xi$. We do so by calculating the correlation function $\left\langle m_{\alpha}(\mathbf{k}) m_{\alpha}(-\mathbf{k})\right\rangle$, where $m_{\alpha}(\mathbf{r})=\int_{d n} \theta(n) \delta\left[\mathbf{r}_{\alpha}(n)-\mathbf{r}\right]$ is the order parameter 
that measures microphase segregation; $\mathbf{k}$ is the Fourier variable conjugate to space dimension. In order to compute this correlation function we follow the method employed by Sfatos et al. ${ }^{16}$ to calculate this quantity for uncorrelated sequence fluctuations. We add the following source term to the Hamiltonian:

$$
\sum_{\alpha} \int d r \int d n \theta(n) \delta\left[r_{\alpha}(n)-r\right] .
$$

Then, we compute the $m$-replica partition function by computing a Gaussian integral related to that in Eq. (5) for the energy. Taking two functional derivatives of the $m$-replica partition function with respect to the source field, noting that we are considering compact states, and renormalizing the energy scale [Eq. (8)] to reflect the fact that we are interested in microphase segregation on the scale of the correlation length $\xi$ yields an expression for $\left\langle m_{\alpha}(\mathbf{k}) m_{\alpha}(-\mathbf{k})\right\rangle$. This expression is identical to Eq. (3.13) in Reference 16, except that the energy scale is renormalized according to Eq. (8). The temperature $\left(T_{\mathrm{mps}}\right)$ at which this correlation function diverges corresponds to the one at which microphase segregation occurs on the scale $\xi$. Our simple calculation shows that $T_{\mathrm{mps}}$ scales with $\xi$ as follows:

$$
T_{\mathrm{mps}} \sim \sqrt{\xi} .
$$

\section{DISCUSSION}

The main result of this paper is the scaling of $T_{f}$ with $\xi$. Eq. (13) shows that the temperature below which a few dominant conformations (on the scale $\xi$ ) determine the thermodynamics grows with the correlation length. This is so because the factor of $\xi$ in the numerator in Eq. (13) grows much faster than the logarithmic term in the denominator. For the specific case of random block copolymers, practical values of $\xi$ are expected to be such that the logarithmic correction is small, thus, $T_{f}$ will essentially grow as the $\sqrt{ } \xi$.

It is important to note that the phenomenon of freezing that we are discussing occurs on larger scales than in the case of RHPs with uncorrelated sequence fluctuations. In this case, in contrast to the latter situation, there is no conformational freezing on the segmental scale; freezing into a few dominant conformations occurs only on a mesoscopic scale. It may seem then that since we are observing the same phenomenon on larger length scales, it is obvious that the phenomenon will be manifested at higher temperatures. However, the current situation is more delicate. Freezing of RHPs involves a subtle interplay between an energetic driving force that promotes freezing $(b)$ and chain flexibility [e.g., Refs. 16-19]. The more flexible the chains, the lower the temperature at which freezing into a few dominant conformations occurs. In fact, as is well known, ${ }^{16}$ for sufficiently flexible chains microphase segregation pre-empts freezing. When we interrogate the system on the scale of the correlation length, the energetic driving force grows. At the same time, however, it is physically obvious (and our equations demonstrate this too) that the RHPs become effectively more flexible as $\xi$ increases. Thus, at the outset, it is not clear which factor dominates and whether the temperature at which freezing occurs on the scale of the correlation length grows or decreases with $\xi$. Our results show that the renormalization of the energetic driving force beats out the effect of enhanced effective flexibility. Thus, freezing on larger scales occurs at higher temperatures as $\xi$ grows.

This result is of experimental consequence. The phenomenon of freezing is a manifestation of frustrated systems with quenched disorder. RHPs provide an excellent vehicle to study the physics of such systems in detail, much like diblock copolymers have enhanced our knowledge of ordering transitions on mesoscopic scales. Our results show that RHPs with correlated sequence fluctuations (with random block copolymers being a good example) should exhibit the phenomenon of freezing on mesoscopic scales at higher temperatures than RHPs with sequence fluctuations on the segmental scale. This interesting manifestation of frustration and quenched disorder could therefore be experimentally observed more readily by studying random block copolymers, both from the point of view of the temperature to be accessed and the length scale resolution required.

As noted earlier, it is well-known that, ${ }^{16}$ for RHPs with a given chemistry, below a threshold level of chain flexibility the freezing phenomenon pre-empts microphase segregation from occurring. Consider a specific RHP chemistry which is such that when sequence fluctuations occur on the segmental scale $(\xi=1)$ freezing pre-empts microphase segregation (i.e., the RHP is sufficiently stiff). We have shown that $T_{f}$ grows with $\xi$, and freezing occurs on the scale $\xi$. Hence, we argued that studying RHPs with this same chemistry but using random block copolymers would make the phenomenon of freezing experimentally more accessible both from the point of view of the temperature range to be probed and the length scale resolution required of the instrumentation. This is true, however, only if $T_{\mathrm{mps}}$ does not grow much more rapidly with $\xi$, thus pre-empting freezing even if the intrinsic chemistry $(\xi=1)$ favors freezing first. Eqs. (13) and (15) show that $T_{\text {mps }} / T_{f} \sim \sqrt{ } \ln \xi$. The extremely slow growth of this ratio implies that, by using random block copolymers with reasonable values of $\xi, T_{f}$ would remain larger than $T_{\text {mps }}$ if the same was true for $\xi=1$. Pathalogically large values of $\xi$ would be required to make microphase segregation pre-empt freezing into a few dominant conformations in such situations.

In Fig. 1, we schematically depict how the experimentally measurable correlation function, $\left\langle m_{\alpha}(\mathbf{k}) m_{\alpha}(-\mathbf{k})\right\rangle$, varies with temperature. The temperature at which the correlation function becomes a constant due to freezing will be higher for RHPs with correlated sequence fluctuations, and the length scale resolution required to observe this shape is far less stringent.

\section{CONCLUDING REMARKS}

Using replica calculations, we have examined the phenomenon of freezing in RHPs with correlated sequence fluctuations. Specifically, we have studied situations where the correlations decay within a single finite length scale; random block copolymers provide a practical example of such sys- 


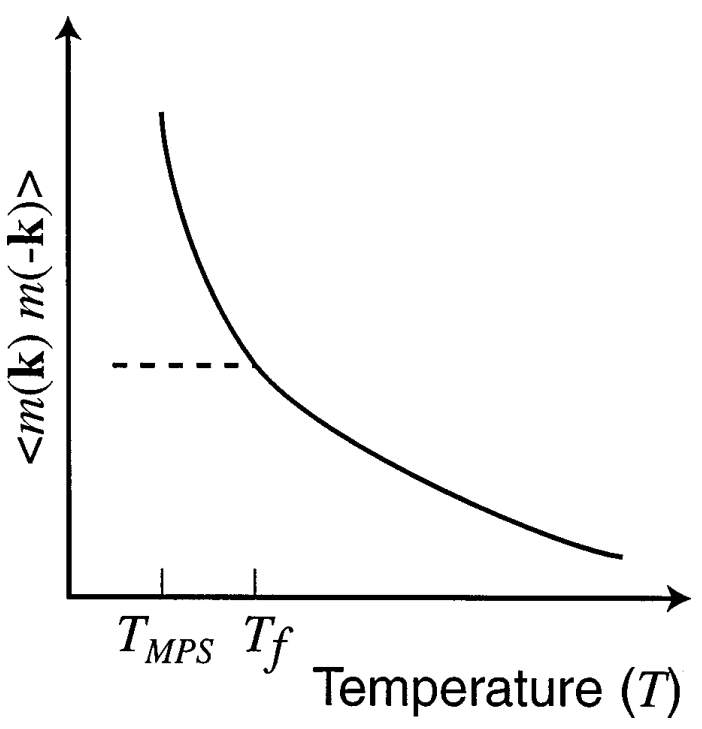

FIG. 1. Sketch of the variation of the correlation function, $\left\langle m_{\alpha}(\mathbf{k}) m_{\alpha}\right.$ $(-\mathbf{k})\rangle$, as a function of temperature. The full line shows the case where microphase segregation occurs. The dotted line shows what happens when freezing pre-empts microphase segregation.

tems. We find that, compared to RHPs with uncorrelated sequence fluctuations, freezing on scales commensurate with the correlation length manifests itself at higher temperatures. Furthermore, if a given chemistry with uncorrelated sequence fluctuations exhibits freezing before microphase segregation, the same will be true for most practical values of the correlation length. These findings suggest that experimental exploration of the phenomenon should be more accessible by using random block copolymers. Furthermore, this phenomenon (a manifestation of frustration and quenched disorder) wherein freezing (not microphase separation) into a few dominant conformations occurs on mesoscopic length scales could lend these materials rather special mechanical properties. For example, the response to deformations on segmental time scales will be liquidlike, but deformations probing time scales commensurate with the scale $\xi$ will lead to interesting dynamical behavior that is difficult to predict currently. We hope that experiments exploring the above issues will be conducted.

\section{ACKNOWLEDGMENT}

Financial support for this work was provided by the U.S. D.O.E., Basic Energy Sciences.

${ }^{1}$ E. I. Shakhnovich and A. M. Gutin, Nature (London) 346, 773 (1990).

${ }^{2}$ P. G. Wolynes, J. N. Onuchic, and D. Thirumalai, Science 267, 1619 (1995)

${ }^{3}$ A. Sali, E. I. Shakhnovich, and M. Karplus, Nature (London) 369, 248 (1994).

${ }^{4}$ H. S. Chan, and K. A. Dill, Phys. Today 46, 24 (1993).

${ }^{5}$ V. S. Pande, A. Y. Grosberg, and T. Tanaka, Proc. Natl. Acad. Sci. USA 91, 12972 (1994)

${ }^{6}$ A. Irback, C. Peterson, and F. Potthast, Proc. Natl. Acad. Sci. USA 93, 9533 (1996)

${ }^{7}$ S. Srebnik, A. K. Chakraborty, and E. I. Shakhnovich, Phys. Rev. Lett. 77, 3157 (1996)

${ }^{8}$ D. Bratko, A. K. Chakraborty, and E. I. Shakhnovich, Chem. Phys. Lett. (in press).

${ }^{9}$ L. Gutman and A. K. Chakraborty, J. Chem. Phys. 101, 10074 (1994).

${ }^{10}$ L. Gutman and A. K. Chakraborty, J. Chem. Phys. 104, 7306 (1996).

${ }^{11}$ J. F. Joanny, J. Phys. II 4, 1281 (1994).

${ }^{12}$ L. Gutman and A. K. Chakraborty, J. Chem. Phys. 103, 10738 (1995).

${ }^{13}$ Y. Lyatska, D. Gersappe, N. A. Gross, and A. C. Balazs, J. Phys. Chem. 100, 1449 (1996).

${ }^{14}$ S. T. Milner and G. H. Fredrickson, Macromolecules 28, 7953 (1995).

${ }^{15}$ T. Garel, D. A. Huse, S. Leibler, and H. Orland, Europhys. Lett. 8, 9 (1989)

${ }^{16}$ C. D. Sfatos, A. M. Gutin, and E. I. Shakhnovich, Phys. Rev. E 48, 465 (1993).

${ }^{17}$ E. I. Shakhnovich and A. M. Gutin, J. Phys. (France) 50, 1843 (1989).

${ }^{18}$ T. Garel and H. Orland, Europhys. Lett. 6, 307 (1988).

${ }^{19}$ E. I. Shakhnovich and A. M. Gutin, Biophys. Chem. 34, 187 (1989).

${ }^{20}$ G. H. Fredrickson, S. T. Milner, and L. Leibler, Macromolecules 25, 6341 (1993)

${ }^{21}$ S. F. Edwards, and P. W. Anderson, J. Phys. F 5, 965 (1975).

${ }^{22}$ V. S. Pande, A. Yu. Grosberg, C. Joerg, and T. Tanaka, Phys. Rev. Lett. 76, 3987 (1996); V. S. Pande, A. Yu. Grosberg, C. Joerg, M. Kardar, and T. Tanaka, ibid. 77, 3565 (1996).

${ }^{23}$ M. Mezard and G. Parisi, J. Phys. (France) 1, 809 (1991).

${ }^{24}$ D. Bratko, A. K. Chakraborty, and E. I. Shakhnovich, Phys. Rev. Lett. 76, 1844 (1996).

${ }^{25}$ K. H. Fischer and J. A. Hertz, Spin Glasses (Cambridge University Press, Cambridge, 1993); M. Mezard, G. Parisi, and M. A. Virasoro, Spin Glass Theory and Beyond (World Scientific, Singapore, 1987).

${ }^{26}$ A. Yu. Grosberg and A. R. Khokhlov, Statistical Physics of Macromolecules (AIP Press, New York, 1994). 